\title{
Budgerigar Testicular Carcinoma
}

National Cancer Institute

\section{Source}

National Cancer Institute. Budgerigar Testicular Carcinoma. NCI Thesaurus. Code C135003.

Carcinoma that arises in the testicle of a budgerigar. 